\title{
Plasma concentrations of angiogenetic factors and angiogenetic inhibitors in patients with ductal pancreatic neoplasms. A pilot study
}

\author{
Raffaele Pezzilli' ${ }^{1}$, Dario Fabbri ${ }^{1}$, Massimiliano $M$. \\ Corsi $^{3}$, Andrea Imbrogno ${ }^{1}$, Alessandra Barassi ${ }^{4}$, \\ Antonio M. Morselli-Labate ${ }^{1}$, Giada Dogliotti ${ }^{3}$, \\ Riccardo Casadei $^{2}$, Roberto Corinaldesi ${ }^{1}$ and \\ Gianvico Melzi d'Eril ${ }^{4}$ \\ ${ }^{1}$ Department of Digestive Diseases and Internal Medicine, \\ Sant'Orsola-Malpighi Hospital, University of Bologna, \\ Bologna, Italy \\ ${ }^{2}$ Department of Emergency, General and Transplant \\ Surgery, Sant'Orsola-Malpighi Hospital, University of \\ Bologna, Bologna, Italy \\ ${ }^{3}$ Institute of General Pathology, University of Milan, \\ Milan, Italy \\ ${ }^{4}$ Department of Medicine, Surgery and Dentistry, \\ University of Milan, Milan, Italy
}

\begin{abstract}
Background: The aim of the study was to evaluate the circulating concentrations of vascular endothelial growth factor (VEGF), vascular endothelial growth factor receptor-2 (VEGFR-2), vascular endothelial growth factor-D (VEGF-D) and endostatin in patients with intraductal papillary mucinous neoplasm (IPMN), and in those with ductal adenocarcinomas. Methods: Sixty patients (32 males, 28 females, mean age $69.3 \pm 11.3$ years) were enrolled: $31(51.7 \%)$ had IPMNs and $29(48.3 \%)$ had histologically confirmed pancreatic adenocarcinomas. Thirty blood donors were also studied as controls. In all study subjects, the concentrations of VEGF, VEGF-D, VEGFR-2, and endostatin were determined using enzyme-linked immunosorbent assays.

Results: Serum concentrations of VEGF, VEGF-D, and VEGFR-2 were significantly higher in patients with pancreatic ductal adenocarcinoma and those with IPMNs compared with healthy subjects, while endostatin was significantly higher only in patients with pancreatic ductal adenocarcinoma compared with healthy subjects. Within the group of patients, VEGFR-2 was significantly higher in patients with ductal adenocarcinoma compared to those with IPMNs. The sensitivity and the specificity of VEGFR-2 in differentiating patients with ductal adenocarcinomas from those with IPMN

\footnotetext{
*Corresponding author: Raffaele Pezzilli, Dipartimento di Malattie Apparato Digerente e Medicina Interna, Ospedale Sant'OrsolaMalpighi, Via Massarenti, 9, 40138 Bologna, Italy

Phone: +39051636 4148, Fax:+39051636 4148,

E-mail: raffaele.pezzilli@aosp.bo.it

Received November 22, 2010; accepted January 4, 2011;

previously published online March 17, 2011
}

at a cut-off range of $4003-4034 \mathrm{pg} / \mathrm{mL}$ was $86.2 \%$ and $54.8 \%$, respectively.

Conclusions: IPMNs have serum VEGFR-2 concentrations different from those in patients with ductal adenocarcinomas. However, serum VEGFR-2 cannot be routinely utilized to differentiate IPMNs from pancreatic ductal adenocarcinomas.

Keywords: angiogenesis inducing agents; endostatins; pancreatic neoplasms; vascular endothelial growth factor receptor-2.

\section{Introduction}

Intraductal papillary mucinous neoplasms (IPMNs) of the pancreas are a group of slow-growing tumors which can be cured surgically in most patients (1). There is little data regarding the inflammation processes associated with this disease. We have recently demonstrated that serum tumor necrosis factor receptor- 1 is elevated in patients with IPMNs and in those with pancreatic adenocarcinomas, suggesting high apoptotic activity in both groups of patients studied (2). Among the causes of the aggressive behavior of ductal exocrine pancreatic tumors, angiogenesis appears to be essential for tumor growth and the development of metastases and angiogenetic factors, such as vascular endothelial growth factor (VEGF), which seem to play a pivotal role (3). VEGF specifically interacts with receptor tyrosine kinases, such as vascular endothelial growth factor receptor-2 (VEGFR-2), which are mainly expressed by endothelial cells, but also by some cancer cells, and they seem to be responsible for the poor prognosis of various tumors (4). Furthermore, vascular endothelial growth factor-D (VEGF-D) is one of the major factors associated with the growth of lymphatic endothelial cells (5). Finally, endostatin is a potent inhibitor of angiogenesis, and increased concentrations of this protein may be associated with cancer progression (6). Thus, several active and passive strategies appear to be adopted by tumor cells to determine the spread of the tumor from the primary site.

All these substances have been studied in pancreatic ductal adenocarcinoma, mainly in pathological specimens, but not in patients with pancreatic IPMNs. Thus, the main aim of the present study was to evaluate the circulating concentrations of VEGF, VEGF-D, VEGFR-2 and endostatin in malignant diseases of the pancreas, such as IPMNs and pancreatic adenocarcinomas. We also evaluated the concentrations of these molecules in comparison to CA 19-9. 


\section{Materials and methods}

\section{Patients}

Consecutive patients, 18 years of age or older, who were admitted to the Unit for the Study of Pancreatic Diseases of Sant'OrsolaMalpighi Hospital of Bologna (Italy) for exocrine pancreatic neoplasms between October 2007 and December 2009 were eligible for inclusion in the study.

A total of 60 patients ( 32 males, 28 females, mean age $69.3 \pm$ 11.3 years) were enrolled: 31 (51.7\%) had IPMNs and $29(48.3 \%)$ had histologically-confirmed pancreatic adenocarcinoma; the demographic and clinical characteristics of the patients studied are reported in Table 1. The body mass index (BMI) was stratified according to the World Health Organization (WHO) classification (7), and pancreatic insufficiency was defined as a fecal elastase- 1 concentration of $<200 \mu \mathrm{g} / \mathrm{g}(8)$. At the time of the study, none of the patients had had any treatment for their disease. Of the 31 patients with IPMNs, 16 (51.6\%) had branch type IPMNs and the remaining $15(48.4 \%)$ had main duct type IPMNs.

As shown in Table 1, the two groups of patients were not statistically different regarding gender $(p=1.000)$, age $(p=0.594)$ and localization of the tumor $(\mathrm{p}=0.166)$ whereas the BMI of the patients with pancreatic ductal adenocarcinoma was significantly lower than that of patients having an IPMN $(\mathrm{p}<0.025)$. In addition, the frequency of metastases $(\mathrm{p}<0.001)$, pain $(\mathrm{p}<0.001)$, jaundice $(p=0.002)$ and diabetes $(p<0.001)$ were significantly higher in patients with pancreatic ductal adenocarcinoma than in those having IPMNs. The frequency of pancreatic exocrine insufficiency at the time of the study, and the frequency of patients who underwent pancreatic surgery after the diagnosis, were not different between the groups of patients studied $(\mathrm{p}=1.000$ and $\mathrm{p}=0.108$, respectively).

Finally, 30 blood donors were also studied as healthy controls (17 males, 13 females; mean age $59.7 \pm 8.1 ; \mathrm{p}=0.825$ and $\mathrm{p}<0.001$ vs. the gender and the age of patients, respectively).

\section{Methods}

Fasting blood specimens were obtained in the morning from each subject enrolled. Laboratory personnel were unaware of the clinical diagnoses or the details of the patients' clinical histories. Serum was stored at $-20^{\circ} \mathrm{C}$ until analysis.

Concentrations of VEGF, VEGF-D, VEGFR-2, and endostatin were determined using enzyme-linked immunosorbent assays (ELISA) (R\&D System, Minneapolis, MN, USA). This analysis employs a quantitative sandwich enzyme immunoassay technique. A monoclonal antibody specific for this molecule was pre-coated onto a microplate. After dilution, standards and samples were pipetted into the wells. After washing away any unbound substances, an enzymelinked monoclonal antibody specific for each of these molecules was added to the wells. After removing any unbound antibodyenzyme reagent, substrate solution was added to the wells and color developed in proportion to the amount of our specific molecules. The reaction was stopped with $50 \mu \mathrm{L} \mathrm{HCl}$ and the intensity of the color was measured at $450 \mathrm{~nm}$. The dilution factor was recorded in order to calculate the concentration of VEGF, VEGF-D, VEGFR-2 and endostatin in the samples.

The maximum intra- and inter-assay coefficients of variation (CVs) were $5.44 \%$ and $7.30 \%$ for VEGF (20 determinations at each

Table 1 Demographic and clinical characteristics of patients with malignant pancreatic diseases according to the final diagnosis (data are reported as mean $\pm \mathrm{SD}$ or frequencies).

\begin{tabular}{|c|c|c|c|}
\hline & $\begin{array}{l}\text { Pancreatic intraductal } \\
\text { papillary mucinous neoplasm } \\
\text { (no. 31) }\end{array}$ & $\begin{array}{l}\text { Pancreatic } \\
\text { adenocarcinoma } \\
\text { (no. 29) }\end{array}$ & p-Value \\
\hline Gender & & & $1.000^{\mathrm{a}}$ \\
\hline Males & $17(54.8 \%)$ & $15(51.7 \%)$ & \\
\hline Females & $14(45.2 \%)$ & $14(48.3 \%)$ & \\
\hline Age, years & $69.8 \pm 10.2$ & $68.7 \pm 12.5$ & $0.594^{\mathrm{b}}$ \\
\hline Body mass index (BMI), $\mathrm{kg} / \mathrm{m}^{2}$ & $23.0 \pm 3.1$ & $21.1 \pm 2.6$ & $<0.025^{\mathrm{b}}$ \\
\hline BMI classes & & & $0.022^{\mathrm{c}}$ \\
\hline Underweight, $<18.5 \mathrm{~kg} / \mathrm{m}^{2}$ & 0 & $5(17.2 \%)$ & \\
\hline Normal, $18.5-24.9 \mathrm{~kg} / \mathrm{m}^{2}$ & $28(90.3 \%)$ & $23(79.3 \%)$ & \\
\hline Preobese, $25-29.9 \mathrm{~kg} / \mathrm{m}^{2}$ & $2(6.5 \%)$ & $1(3.4 \%)$ & \\
\hline Obese, $30 \mathrm{~kg} / \mathrm{m}^{2}$ or more & $1(3.2 \%)$ & 0 & \\
\hline Localization & & & $0.166^{\mathrm{d}}$ \\
\hline Head & $19(61.3 \%)$ & $26(89.7 \%)$ & \\
\hline Head-body & $1(3.2 \%)$ & 0 & \\
\hline Body & $3(9.7 \%)$ & $1(3.4 \%)$ & \\
\hline Body/tail & $1(3.2 \%)$ & $1(3.4 \%)$ & \\
\hline Tail & $4(12.9 \%)$ & $1(3.4 \%)$ & \\
\hline Diffuse & $3(9.7 \%)$ & 0 & \\
\hline Metastases & $2(6.5 \%)$ & $21(72.4 \%)$ & $<0.001^{\mathrm{a}}$ \\
\hline Pain & $8(25.8 \%)$ & $27(93.1 \%)$ & $<0.001^{\mathrm{a}}$ \\
\hline Jaundice & $4(12.9 \%)$ & $15(51.7 \%)$ & $0.002^{\mathrm{a}}$ \\
\hline Diabetes & $11(35.5 \%)$ & $24(82.8 \%)$ & $<0.001^{\mathrm{a}}$ \\
\hline $\begin{array}{l}\text { Pancreatic exocrine insufficiency, } \\
\text { fecal elastase- } 1<200 \mu \mathrm{g} / \mathrm{g}\end{array}$ & $9(29.0 \%)$ & $9(31.0 \%)$ & $1.000^{\mathrm{a}}$ \\
\hline Pancreatic surgery & $8(25.8 \%)$ & $14(48.3 \%)$ & $0.108^{\mathrm{a}}$ \\
\hline
\end{tabular}

${ }^{\mathrm{a}}$ Fisher's exact test. ${ }^{\mathrm{b}} \mathrm{Kruskal}-$ Wallis test. ${ }^{\mathrm{C}}$ Linear-by-linear association $\chi^{2}$. ${ }^{\mathrm{d} P e a r s o n} \chi^{2}$. 
Table 2 Circulating concentrations of the various substances studied in the three groups of subjects. Data are reported as mean \pm SD.

\begin{tabular}{|c|c|c|c|c|}
\hline & $\begin{array}{l}\text { Pancreatic intraductal } \\
\text { papillary mucinous } \\
\text { neoplasm (no. } 31 \text { ) }\end{array}$ & $\begin{array}{l}\text { Pancreatic } \\
\text { adenocarcinoma } \\
\text { (no. 29) }\end{array}$ & p-Value ${ }^{a}$ & $\begin{array}{l}\text { Healthy } \\
\text { subjects } \\
\text { (no. 30) }\end{array}$ \\
\hline VEGF, pg/mL & $1210 \pm 1004$ & $1175 \pm 664$ & 0.348 & $328 \pm 173$ \\
\hline p vs. healthy subjects & $\mathrm{p}<0.001$ & $\mathrm{p}<0.001$ & & - \\
\hline VEGF-D, pg/mL & $854 \pm 493$ & $878 \pm 500$ & 0.994 & $342 \pm 58$ \\
\hline p vs. healthy subjects & $\mathrm{p}<0.001$ & $\mathrm{p}<0.001$ & & - \\
\hline VEGFR-2, pg/mL & $4216 \pm 794$ & $5031 \pm 1142$ & 0.006 & $2313 \pm 722$ \\
\hline p vs. healthy subjects & $\mathrm{p}<0.001$ & $\mathrm{p}<0.001$ & & - \\
\hline Endostatin, $\mathrm{ng} / \mathrm{mL}$ & $79.4 \pm 61.4$ & $128.9 \pm 105.4$ & 0.058 & $46.9 \pm 17.1$ \\
\hline p vs. healthy subjects & $\mathrm{p}=0.108$ & $\mathrm{p}<0.001$ & & - \\
\hline CA $19-9, \mathrm{U} / \mathrm{mL}$ & $776 \pm 3686$ & $1206 \pm 2450$ & 0.002 & $11 \pm 8$ \\
\hline p vs. healthy subjects & $\mathrm{p}=0.014$ & $\mathrm{p}<0.001$ & & - \\
\hline
\end{tabular}

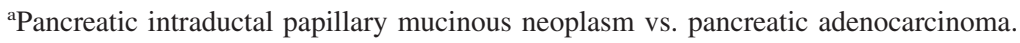

concentration of 50,250 and $900 \mathrm{pg} / \mathrm{mL}), 4.20 \%$ and $7.47 \%$ for VEGF-D (20 determinations at each concentration of 250, 900 and $2000 \mathrm{pg} / \mathrm{mL}$ ), $3.57 \%$ and $6.54 \%$ for VEGFR-2 (20 determination at each concentration of 2000,5000 and $10,000 \mathrm{pg} / \mathrm{mL}), 5.50 \%$ and $6.57 \%$ for endostatin (20 determination at each concentration of 10 , 50 and $100 \mathrm{ng} / \mathrm{mL}$ ), respectively. The detection limits were $9 \mathrm{pg}$ / $\mathrm{mL}$ for VEGF, $11.4 \mathrm{pg} / \mathrm{mL}$ for VEGF-D, $7 \mathrm{pg} / \mathrm{mL}$ for VEGFR-2, and $0.023 \mathrm{ng} / \mathrm{mL}$ for endostatin. Finally, we also assayed CA 19-9 using an electrochemical luminescence immunoassay (CA 19-9, Roche, Milan, Italy; reference limits $0-37 \mathrm{U} / \mathrm{mL}$ ).

\section{Ethics}

The study was approved by the Clinical Committee of the Department of Internal Medicine of Sant'Orsola Hospital of Bologna (Italy) and was performed in accordance with the Helsinki Declaration of the World Medical Association. All subjects gave written informed consent to participate in the study.

\section{Statistical analysis}

Means, standard deviations (SD), and frequencies were used as descriptive statistics. Data were analyzed by means of non-parametric tests: the Kruskal-Wallis test, the Fisher's exact test, the Pearson $\chi^{2}$, and the linear-by-linear association $\chi^{2}$. The forward multivariate linear regression was used in order to identify the clinical variables independently related to the various analytes studied. Receiver operating characteristic (ROC) curve analysis was used to differentiate patients with pancreatic adenocarcinomas from those with pancreatic IPMNs. The best cut-off values were chosen to be the values of the assays which maximized the likelihood ratio (LR) obtained using the following formula: $L R=$ (Probability of true positive + Probability of true negative)/(Probability of false positive + Probability of false negative) (9). The SPSS (SPSS Inc., Chicago, IL, USA, Version 13.0) statistical package was used to analyze the data. Two-tailed p-values of $<0.05$ were considered statistically significant.

\section{Results}

The mean $\pm S D$ values of VEGF, VEGF-D, VEGFR-2, endostatin and CA 19-9 in the three groups of patients studied are reported in Table 2.

Serum concentrations of VEGF, VEGF-D, VEGFR-2 and CA 19-9 were significantly higher both in patients with pancreatic ductal adenocarcinoma and in those with IPMN compared with healthy subjects, while endostatin was significantly higher in pancreatic ductal adenocarcinoma patients only compared with healthy subjects. Within the group of patients, VEGFR-2 and CA 19-9 were significantly higher in patients with pancreatic ductal adenocarcinoma than in those with IPMN, while no significant differences were observed for VEGF, VEGF-D and endostatin. For this reason, we calculated the best cut-off vales for both VEGFR-2 and CA 19-9 in order to differentiate patients with pancreatic ductal adenocarcinoma from those with IPMN. As reported in Table 3, the area under the curve (AUC) and the standard error of AUC (SE) for VEGFR-2 and CA 19-9 were $0.707 \pm 0.067$ and $0.727 \pm 0.070$, respectively. Using a cutoff range of 4003-4034 pg/mL for VEGFR-2, the sensitivity and the specificity of this chemokine in differentiating patients with pancreatic adenocarcinoma from those with IPMN was $86.2 \%$ and $54.8 \%$, respectively, while, at a cutoff range of 63-89 U/mL, CA 19-9 had a sensitivity of $72.4 \%$ and a specificity of $74.2 \%$.

Table 3 Area under the curve (AUC) and standard error of AUC (SE) for VEGFR-2 and CA 19-9 for differentiating patients with pancreatic adenocarcinoma from those with pancreatic intraductal papillary mucinous neoplasms (IPMNs). Sensitivity and specificity were evaluated at the best cut-off range.

\begin{tabular}{lllll}
\hline & AUC \pm SE & Sensitivity, $\%$ & Specificity, $\%$ & Cut-off range \\
\hline VEGFR-2 & $0.707 \pm 0.067$ & 86.2 & 54.8 & $4003-4034 \mathrm{pg} / \mathrm{mL}$ \\
CA 19-9 & $0.727 \pm 0.070$ & 72.4 & 74.2 & $63-89 \mathrm{U} / \mathrm{mL}$ \\
\hline
\end{tabular}


Regarding the clinical variables, the 23 patients with metastasis (two patients with IPMN and 21 patients with pancreatic adenocarcinoma) had serum concentrations of VEGFR-2, endostatin and CA 19-9 that were significantly higher (VEGFR-2: $5107 \pm 1216 \mathrm{pg} / \mathrm{mL}, \mathrm{p}=0.012$; endostatin: $131.0 \pm 98.2 \mathrm{ng} / \mathrm{mL}, \mathrm{p}=0.046$; CA 19-9: $2163 \pm 4852$ $\mathrm{U} / \mathrm{mL}, \mathrm{p}=0.015)$ than the 37 patients without metastasis (VEGFR-2: $4302 \pm 810 \mathrm{pg} / \mathrm{mL}, \mathrm{p}=0.012$; endostatin: $86.1 \pm$ $78.2 \mathrm{ng} / \mathrm{mL}, \mathrm{CA} 19-9: 251 \pm 492 \mathrm{U} / \mathrm{mL}$ ), whereas the 35 patients with diabetes (11 patients with IPMN and 24 patients with pancreatic adenocarcinoma) had serum concentrations of endostatin and CA 19-9 significantly higher (endostatin: $125.1 \pm 100.3 \mathrm{ng} / \mathrm{mL}, \mathrm{p}=0.031$; CA 19-9: $1557 \pm$ $4017 \mathrm{U} / \mathrm{mL}, \mathrm{p}=0.007$ ) than the 25 patients without diabetes (endostatin: $72.8 \pm 57.2 \mathrm{ng} / \mathrm{mL}, \mathrm{CA} 19-9$ : $181 \pm 296 \mathrm{U} / \mathrm{mL}$ ). In addition, CA 19-9 was significantly higher in the 35 patients (eight patients with IPMN and 27 patients with pancreatic adenocarcinoma) with pain $(1019 \pm 2265 \mathrm{U} / \mathrm{mL}) \mathrm{vs}$. the 25 patients without pain $(934 \pm 4102 \mathrm{U} / \mathrm{mL}, \mathrm{p}=0.026)$.

Multivariate analysis showed that the type of the tumor (IPMN or pancreatic adenocarcinoma) only was significantly related to VEGFR-2 serum concentrations $(\mathrm{p}=0.002)$. Also, the presence of diabetes only was significantly related to endostatin serum concentrations $(p=0.022)$. In contrast, no clinical variables entered the analyses for VEGF, VEGF-D. In contrast, the presence of metastases $(p=0.002)$ and jaundice $(\mathrm{p}=0.024)$ were independently and significantly related to CA 19-9 serum concentrations.

Finally, within the group of 31 patients with IPMN, no significant differences in serum concentrations of VEGF, VEGF-D, VEGFR-2, and endostatin were found between the 15 patients with main duct type IPMN and the 16 patients with branch type IPMN (Table 4).

\section{Discussion}

Patients with pancreatic adenocarcinoma have a poor outcome, while those with IPMN of the pancreas have slow growing neoplasms which can be cured surgically in most patients (1). The differences between the two neoplasms are also confirmed by the clinical data of our study. In fact, patients with ductal adenocarcinoma had a frequency of pain, diabetes, jaundice and metastases significantly higher than those with IPMN, as well as having significantly lower BMI. At present, there are no data regarding the angiogenetic fac- tors released by pancreatic IPMNs. Thus, we performed the present study in order to evaluate VEGF, VEGF-D, VEGFR-2 and endostatin in patients with malignant chronic diseases of the pancreas, such as pancreatic adenocarcinoma and IPMN, because a better understanding of the circulating concentrations of angiogenetic and antiangiogenetic factors would be helpful in developing immunotherapeutic approaches to pancreatic neoplasms (10) since there is a lack of conventional immune therapeutic options for these patients, especially for those having IPMN.

For the first time, we comparatively evaluated serum concentrations of angiogenetic and antiangiogenetic factors in patients with pancreatic ductal adenocarcinoma and in patients with pancreatic IPMN. We found that serum concentrations of VEGF, VEGF-D, VEGFR-2 and endostatin were significantly higher in patients with ductal adenocarcinoma than in healthy subjects, and these results are similar to those previously reported for VEGF (3) and endostatin alone (6). Furthermore, data on VEGF-D and VEGFR-2 have previously been reported only on pancreatic tissue and we now report these data for blood. Tumor angiogenesis is often the consequence of an angiogenetic imbalance in which proangiogenetic factors predominate over antiangiogenetic factors (11). The fact that we found that all the circulating angiogenetic factors are increased seems to support the hypothesis that there is an inappropriate response to tumor invasiveness by the host. This is also supported by the fact that VEGFR-2, which is a marker of poor prognosis in various tumors including pancreatic cancer $(4,6)$, shows high serum concentrations in patients with pancreatic ductal adenocarcinoma rather than in those with IPMNs. This is also supported by the finding that patients with advanced cancer, i.e., those with metastasis and those with diabetes, had high serum concentrations of this protein. Multivariate analysis showed the type of tumor (IPMN or pancreatic adenocarcinoma) was the only clinical variable independently related to VEGFR-2 serum concentrations. However, in clinical practice, VEGFR-2 cannot be utilized as a marker to distinguish patients with pancreatic ductal adenocarcinomas from those with IPMN, because even if the sensitivity was $86.2 \%$, the specificity was quite low (54.8\%).

Also, of interest, is the fact that considering only patients with IPMN, those patients having main duct type had similar values of both proangiogenetic and antiangiogenetic factors compared with patients with branch type IPMN.

Regarding CA 19-9, this marker is also not useful for differentiating patients with pancreatic ductal adenocarcinoma

Table 4 Circulating concentrations of the various substances studied in the two groups of patients with pancreatic intraductal papillary mucinous neoplasms (IPMNs). Data are reported as mean \pm SD.

\begin{tabular}{llll}
\hline & $\begin{array}{l}\text { IPMN main duct type } \\
\text { (no. 15) }\end{array}$ & $\begin{array}{l}\text { IPMN branch type } \\
\text { (no. 16) }\end{array}$ & p-Value \\
\hline VEGF, pg/mL & $1102 \pm 1150$ & $1311 \pm 871$ & 0.089 \\
VEGF-D, pg/mL & $760 \pm 285$ & $941 \pm 628$ & 0.607 \\
VEGFR-2, pg/mL & $4204 \pm 834$ & $4227 \pm 781$ & 0.843 \\
Endostatin, ng/mL & $88.4 \pm 60.1$ & $71.0 \pm 63.3$ & 0.323 \\
CA $19-9, \mathrm{U} / \mathrm{mL}$ & $1483 \pm 5292$ & $113 \pm 241$ & 0.220 \\
\hline
\end{tabular}


from those with IPMN because the sensitivity and specificity were quite low at $72.4 \%$ and $74.2 \%$, respectively. Our data confirm previous studies suggesting that CA 19-9 determination is not recommended for use as a screening test for pancreatic neoplasia $(12,13)$ and, in particular, the multivariate analysis demonstrates that the presence of metastases and jaundice were independently related to CA 19-9 serum concentrations.

In conclusion, our data further suggest that an IPMN is one of the precursors of ductal adenocarcinoma, and it has a pattern of angiogenetic factors different from ductal adenocarcinoma. However, these markers, such as CA 19-9, cannot be utilized to routinely differentiate IPMNs from pancreatic ductal adenocarcinomas.

\section{Conflict of interest statement}

Authors' conflict of interest disclosure: The authors stated that there are no conflicts of interest regarding the publication of this article.

Research funding: None declared.

Employment or leadership: None declared.

Honorarium: None declared.

\section{References}

1. Fritz S, Warshaw AL, Thayer SP. Management of mucin-producing cystic neoplasms of the pancreas. Oncologist 2009;14: 125-36.

2. Pezzilli R, Corsi MM, Barassi A, Morselli-Labate AM, Dogliotti $\mathrm{G}$, Casadei $\mathrm{R}$, et al. The role of inflammation in patients with intraductal mucinous neoplasm of the pancreas and in those with pancreatic adenocarcinoma. Anticancer Res 2010;30:3801-5.

3. Kobayashi A, Yamaguchi T, Ishihara T, Ohshima T, Baba T, Shirai $\mathrm{Y}$, et al. Usefulness of plasma vascular endothelial growth factor in the diagnosis of pancreatic carcinoma: differential diagnosis, tumor progression, and patient survival. Pancreas 2005;31:74-8.

4. Doi Y, Yashiro M, Yamada N, Amano R, Ohira G, Komoto M, et al. Significance of phospho-vascular endothelial growth factor receptor-2 expression in pancreatic cancer. Cancer Sci 2010;101:1529-35.

5. Zhang B, Zhao WH, Zhou WY, Yu WS, Yu JM, Li S. Expression of vascular endothelial growth factors-C and -D correlate with evidence of lymphangiogenesis and angiogenesis in pancreatic adenocarcinoma. Cancer Detect Prev 2007;31:436-42.

6. Ohlund D, Ardnor B, Oman M, Naredi P, Sund M. Expression pattern and circulating levels of endostatin in patients with pancreas cancer. Int J Cancer 2008;122:2805-10.

7. World Health Organization. Obesity: preventing and managing the global epidemic. Report of a WHO consultation. World Health Organ Tech Rep Ser 2000;894:i-xii, 1-253.

8. Pezzilli R, Barassi A, Morselli-Labate AM, Fantini L, Tomassetti P, Campana D, et al. Fecal calprotectin and elastase 1 determinations in patients with pancreatic diseases: a possible link between pancreatic insufficiency and intestinal inflammation. J Gastroenterol 2007;42:754-60.

9. Pezzilli R, Billi P, Miniero R, Fiocchi M, Cappelletti O, Morselli-Labate AM, et al. Serum interleukin-6, interleukin-8, and beta 2-microglobulin in early assessment of severity of acute pancreatitis. Comparison with serum C-reactive protein. Dig Dis Sci 1995;40:2341-8.

10. Ranieri G, Patruno R, Ruggieri E, Montemurro S, Valerio P, Ribatti D. Vascular endothelial growth factor (VEGF) as a target of bevacizumab in cancer: from the biology to the clinic. Curr Med Chem 2006;13:1845-7.

11. Korc M. Pathways for aberrant angiogenesis in pancreatic cancer. Mol Cancer 2003;2:8.

12. Pezzilli R, Casadei R, Calculli L, Santini D, Morselli-Labate AM. Serum determination of CA 19-9 in diagnosing pancreatic cancer: an obituary. Dig Liver Dis 2010;42:73-4.

13. Plebani M, Basso D, Panozzo MP, Fogar P, Del Favero G, Naccarato $\mathrm{R}$. Tumor markers in the diagnosis, monitoring and therapy of pancreatic cancer: state of the art. Int J Biol Markers 1995;10:189-99. 